\title{
Flow-Mediated Interaction between Graphene Oxide Nanosheets and Polycarbonate Chains
}

Xiangfei Chen, Lei Xia, Yajiang Huang*, Yadong Lv, Miqiu Kong, Guangxian Li ${ }^{*}$

College of polymer science and engineering, State key laboratory of polymer materials engineering of China, Sichuan University, Chengdu 610065, China

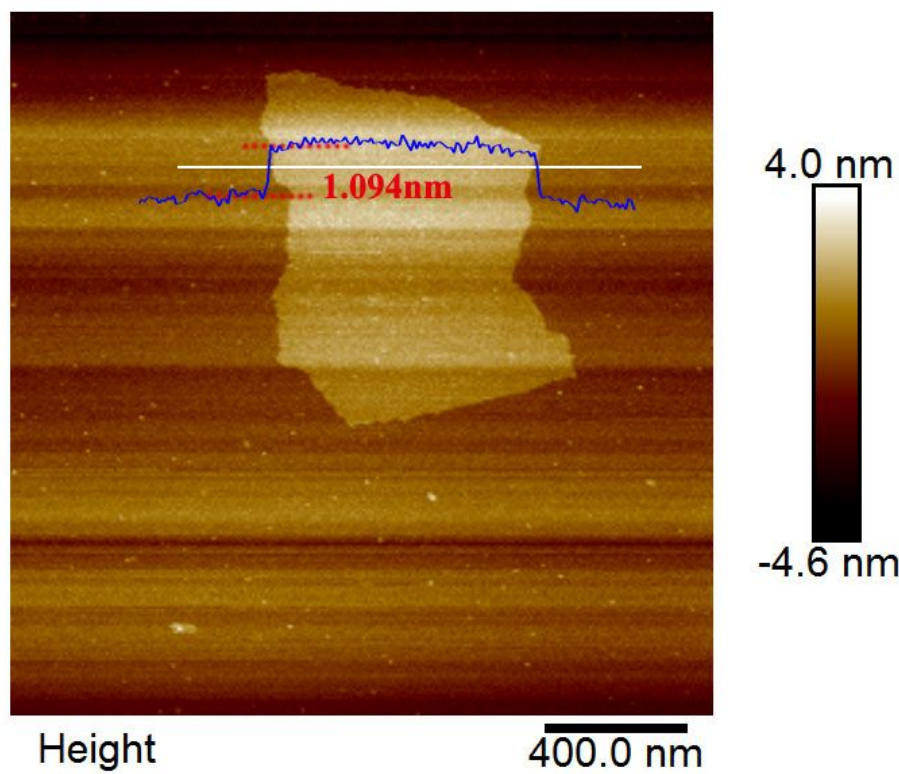

Figure. S 1 AFM image of as-prepared GO.

*Corresponding author. E-mail: hyj@scu.edu.cn; guangxianli@scu.edu.cn. 


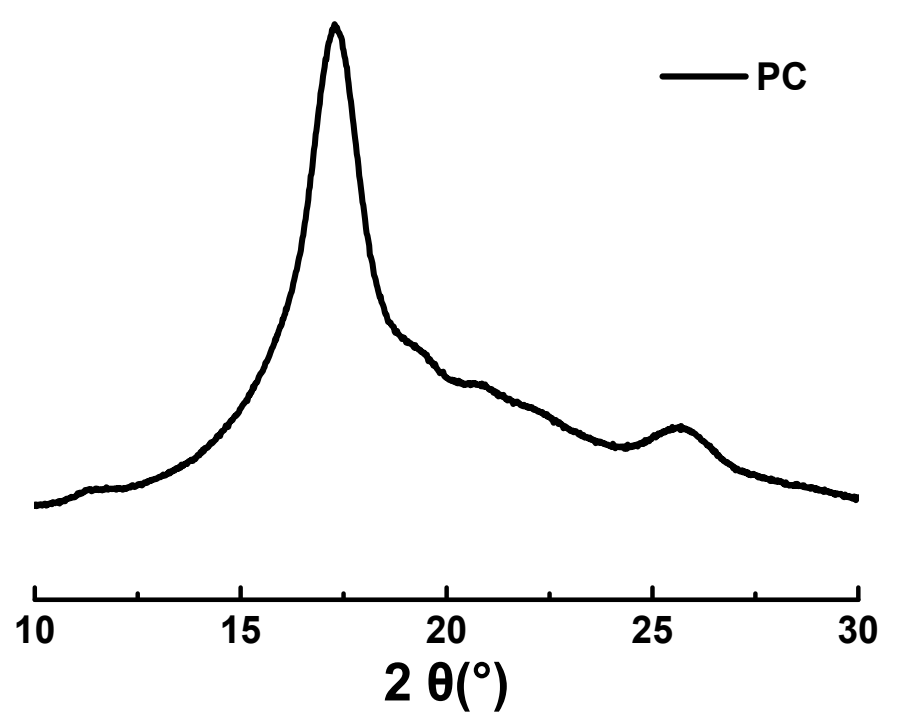

Figure. S 2 X-ray diffraction curve of solution-casted PC.
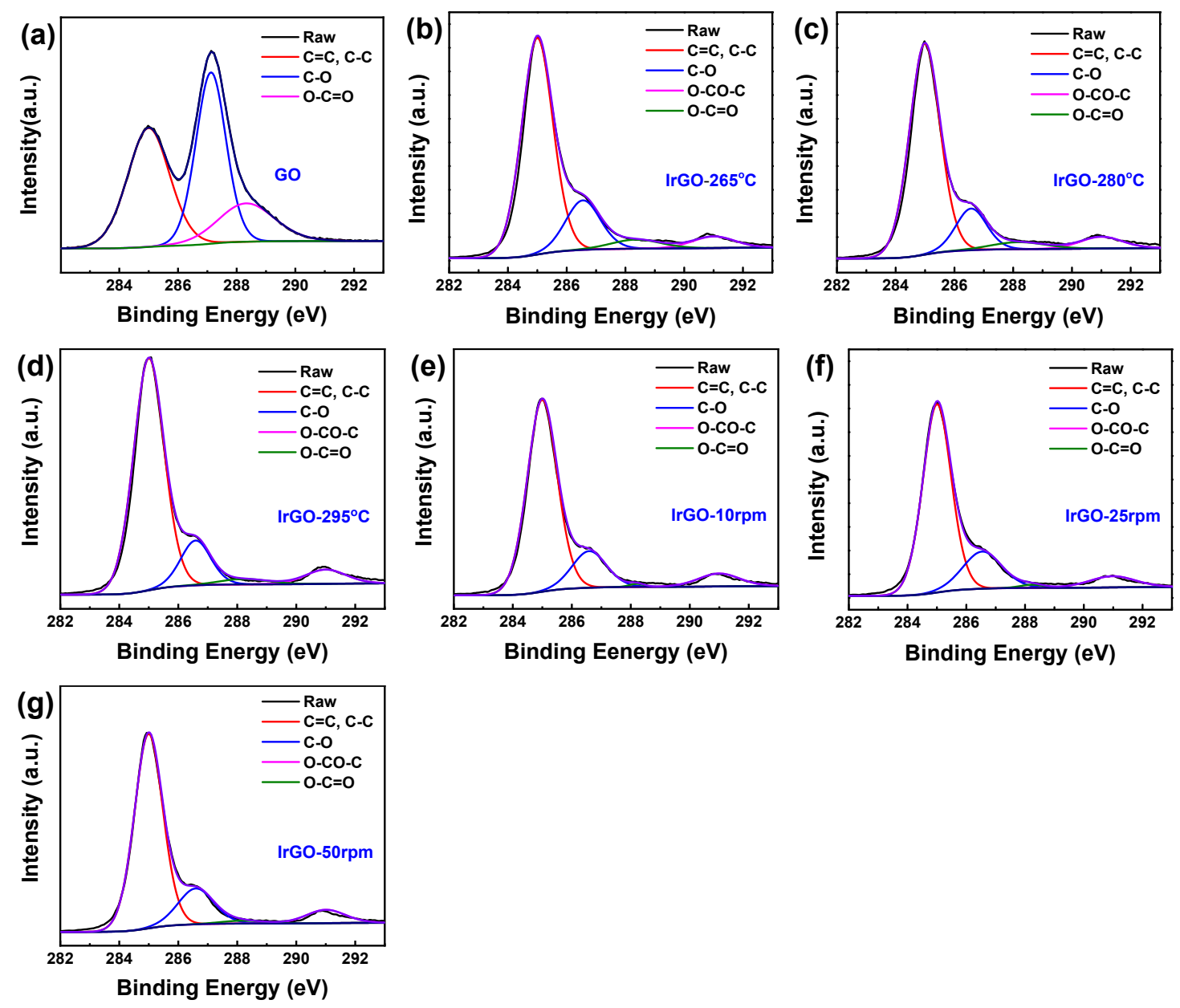

Figure. S 3 Deconvolution of the C 1s XPS peaks of GO and IrGO. 